\title{
Tabaquismo en pacientes hospitalizados
}

\author{
M. A. NIETO GARCÍA, L. ABDEL-KADER MARTÍN, M. M. ROSADO MARTÍN ${ }^{1}$, \\ A. CARRIAZO PÉREZ DE GUZMÁN', J. L. ARIAS JIMÉNEZ²
}

Facultad de Medicina. Universidad de Sevilla. ${ }^{1}$ Servicios Centrales. Servicio Andaluz de Salud, Junta de Andalucía. ${ }^{2}$ Hospital Universitario Virgen Macarena. Servicio Andaluz de Salud. Sevilla.

\author{
SMOKING OF IN-PATIENTS
}

\section{RESUMEN}

Objetivo: Este estudio investiga los conocimientos y las actitudes respecto al hábito de fumar en pacientes hospitalizados. Se pretende así identificar aquellas actuaciones sanitarias que podrían ayudarnos a reducir la prevalencia del hábito tabáquico.

Material y métodos: Estudio transversal de una muestra aleatoria simple de 395 pacientes, del Hospital Universitario Virgen Macarena de Sevilla, de los que 345 (89\%) aceptaron participar en esta investigación. El cuestionario sobre hábito tabáquico, administrado por un sólo encuestador, se complementó con información sobre características epidemiológicas y clínicas de los pacientes.

Resultados: Un $60 \%$ de pacientes son fumadores actuales ò ex-fumadores, con diferencias estadísticamente significativas en la distribución del hábito de fumar según edad, género, educación, nivel socioeconómico y diagnóstico de ingreso. Más del 90\% de los pacientes conoce la relación causal entre tabaco y cáncer de pulmón o enfermedad pulmonar obstructiva crónica, pero esta proporción es menor para las localizaciones oncológicas de laringe (86\%), esófago (86\%) y cavidad oral $(81 \%)$, cardiopatía coronaria $(82 \%)$ y recién nacidos de bajo peso $(78 \%)$ sin que existan diferencias significativas entre fumadores, ex fumadores y no fumadores. El $45 \%$ de los fumadores actuales tenían menos de 15 años cuando comenzaron a fumar y un $40 \%$ adicional entre 15 y 19 años. El $46 \%$ de los fumadores han intentado, sin éxito, dejar este hábito, a pesar de recibir recomendaciones, en este sentido, del personal sanitario (61\%), familiares y amigos $(60 \%)$.

Conclusiones: La actuaciones sanitarias contra el tabaquismo deben contemplar, el apoyo farmacológico y psicoterapéutico a los fumadores actuales ya que una proporción importante de los mismos aunque desean abandonar el tabaquismo, no lo consiguen.

PALABRAS CLAVE: Tabaquismo. Tratamiento farmacológico. Prevención secundaria.
ABSTRACT

Objective: To asses the knowledge and attitude about smoking habit of in-patients in order to detect the sanitary activities that could help to decrease the prevalence of smoking.

Material and methods: Cross-sectional observational study of random sample ( $n=395)$ of in-patients of University Hospital Virgen Macarean, Seville, Spain. Participation rate was $89 \%(n=350)$ and the interviewer-administered questionnaire was completed with epidemiological and clinical additional data.

Results: Sixty per cent of in-patients were smokers or former smokers. There were statistically significant differences in smoking distribution by age, gender, education, socio-economic level and admission diagnosis. Etiologic relationships between tobacco smoking and lung cancer or chronic lung disease were known by more than $90 \%$ of inpatients but other smoking's health effects were not so well known: larynx (86\%), oesophagus (86\%) and oral cavity (81\%) cancer, coronary heart disease (82\%) and low birthweight (78\%). Forty-five per cent of smokers began to smoke before the age of 15 and an additional $40 \%$ at 15-19 year. Forty-six per cent of smokers tried to leave this habit without success in spite of receiving medical advice of leaving smoking $(61 \%)$ and the recommendations of family and friends $(60 \%)$.

Conclusions: Preventive campaigns against tobacco smoking must include pharmacological and psicological treatment for current smokers because an important proportion of them try to leave this habit without success.

KEY WORDS: Smoking. Pharmacological treatment. Secondary prevention.

Nieto García MA, Abdel-Kader Martín L, Rosado Martín MM, Carriazo Pérez de Guzmán A, Arias Jimenez JL. Tabaquismo en pacientes hospitalizados. An Med Interna (Madrid) 2003; 20: 351-359.

\section{INTRODUCCIÓN}

En nuestro país el hábito de fumar se mantiene en cifras elevadas de prevalencia (1) a pesar de que su relación primero con el cáncer de pulmón y más tarde con los cánceres de larin- ge, faringe, cavidad oral, esófago, vejiga, páncreas y riñón está bien acreditada (2-6). Y a dichas relaciones se le añade su importancia como factor de riesgo de patología cardiovascular, enfermedad pulmonar obstructiva crónica y úlcera duodenal (7) con las consiguientes repercusiones sobre la mortali-

Trabajo aceptado: 7 de marzo de 2003 
dad específica por estas causas y general por el conjunto de causas de defunción, así como su participación en la aparición de bajo peso en los nacidos de madres fumadoras (8).

Sin duda se ha insistido más en comprobar sus efectos nocivos en el terreno científico (9) que en transmitir de forma efectiva las conclusiones de estos estudios a la población. Por otra parte, los profesionales de la sanidad y la docencia han mantenido durante décadas prevalencias de tabaquismo elevadas, dificultando las actuaciones preventivas y de control de este hábito. Los médicos fumadores no solían abordar el tabaquismo de sus pacientes y los maestros que fuman no pueden ejercer un rol ejemplar (10). Las corporaciones que agrupan a los profesionales sanitarios, mejor informados que la población general acerca de los efectos perjudiciales sobre la salud del tabaco, no han ejercido ningún papel preventivo ni han estimulado la intervención de la administración. No es extraño, por tanto, que sólo en los últimos años se hayan adoptado en nuestro país algunas de las medidas legislativas recomendadas en el ámbito internacional (11) para reducir el impacto del tabaquismo sobre la salud humana, como la delimitación de áreas de no fumadores, las restricciones a la publicidad del tabaco y la prohibición de fumar en edificios públicos, especialmente centros sanitarios y educativos.

Recientemente se ha comenzado a ofrecer apoyo sanitario al grupo de población, cada vez más numeroso, que desea dejar de fumar (12), una vez convencido de los desvastadores efectos que este hábito tiene sobre la salud $(13,14)$. Se han analizado las características de los que consiguen abandonar el tabaco con éxito (15) para orientar las políticas preventivas y corregir las predicciones que se realizan respecto a la evolución de las enfermedades asociadas al consumo de cigarrillos $(16,17)$.

En otros países se han observado modificaciones importantes de las características de la población fumadora. Así, en EE.UU. entre 1975 y 1984 el nivel educativo se convirtió en el principal predictor del hábito de fumar -prevalencia doble en varones de menor nivel educativo- y del abandono del hábito, que aumentó en todos los niveles educativos pero con un incremento superior al doble en varones de mayor nivel educativo (18).

Este estudio presenta un análisis descriptivo del hábito de fumar, su distribución y características, en pacientes hospitalizados, en un intento de aproximarnos a los motivos del mantenimiento de cifras elevadas de prevalencia de tabaquismo en nuestro medio.

\section{MATERIAL Y MÉTODOS}

Entre el 19 de octubre de 2000 y el 20 de enero de 2001 se seleccionó semanalmente al azar por muestreo aleatorio simple a pacientes ingresados en el Hospital Universitario Virgen Macarena de Sevilla hasta un total de 395 pacientes. Se definieron como criterios de exclusión: edad $<18$ años, una fecha de ingreso superior a una semana -para evitar un exceso de pacientes de larga estancia-, y diagnóstico de ingreso por patologías que impidieran responder con razonable fiabilidad a un cuestionario administrado por una encuestadora, i.e. demencia senil, pacientes postquirúrgicos con sondaje nasogástrico, pacientes en cuidados intensivos y unidades de reanimación, etc.

A todos los pacientes seleccionados, una encuestadora les propuso que respondieran a un cuestionario de 68 items que en los apartados de conocimientos y hábito tabáquico utilizaba una versión reducida del Behavioral Risk Factor Surveillance System cuestionario (19). Se consideró fumador -actual o pasado- a todo paciente que hubiera fumado al menos 100 cigarrillos a lo largo de su vida (20) y ex fumador a quienes declaraban haber abandonado dicho hábito. De los 395 pacientes contactados, 350 aceptaron contestar la encuesta (89\%) mientras que 45 pacientes $(11 \%)$ rechazaron participar en el estudio. Esta muestra supone aproximadamente el $1 \%$ de las altas anuales de este Hospital (21).

El diagnóstico de ingreso se codificó según la lista abreviada de la $10^{\text {a }}$ revisión de la Clasificación Internacional de Enfermedades y Causas de Defunción (CIE-10) (22). El lugar de residencia se codificó utilizando la clasificación de municipios del Instituto de Estadística de Andalucía (IEA), aunque más tarde se agrupó en tres amplios estratos: Sevilla municipio, resto de Sevilla provincia y otros municipios. La ocupación se codificó utilizando la Clasificación Nacional de Ocupaciones (CON-1994) y en función de ésta se establecieron los niveles socioeconómicos, siguiendo la correspondencia entre las ocupaciones a nivel del tercer dígito del anexo $\mathrm{D}$ del informe sobre "Medición de la clase social en Ciencias de la Salud" elaborado y publicado por la Sociedad Española de Epidemiología (23).

Se creó una base de datos con la información recogida de todos los pacientes usando el programa ACCESS, que se capturó y analizó posteriormente con la versión 10.0 del programa SPSS (24). La significación estadística de las diferencias en la distribución por hábito de fumar según edad, género, años de educación, máximo nivel educativo completado, nivel socioeconómico, lugar de residencia y diagnóstico de ingreso se testaron al nivel de confianza del $95 \%$ usando la prueba de chi cuadrado para variables categóricas (25). Las significación estadística de las diferencias en la distribución por hábito de fumar de los conocimientos, percepciones y actuaciones respecto al tabaquismo se testaron al nivel de confianza del 95\% usando la prueba de tendencia lineal para variables con categorías ordenadas y respuesta binaria (26) siempre que los requisitos cuantitativos de aplicación del test de chi cuadrado se incumplían (frecuencias observadas inferiores a cinco). En ambos casos se compararon las categorías de respuestas afirmativas y negativas, tras excluir los efectivos que contestaban "no se" o "no recuerdo".

\section{RESULTADOS}

La tabla I muestra la distribución de la muestra según el hábito de fumar, la edad -en cuartiles aproximados-, el género, los años de educación recibida, el máximo nivel educativo completado, el nivel socioeconómico -clasificado a través de la ocupación-, el lugar de residencia y el diagnóstico de ingreso.

El $60 \%$ de los pacientes fuman actualmente $(27 \%)$ o han fumado (33\%) al menos 100 cigarrillos a lo largo de su vida. Observamos diferencias significativas en la distribución por hábito de fumar según la edad ( $\mathrm{p}<0,001)$, el género $(\mathrm{p}<$ $0,001)$, los años de enseñanza recibidos $(\mathrm{p}=0,002)$, el nivel educativo completado $(\mathrm{p}=0,007)$, el nivel socioeconómico ( $\mathrm{p}$ $=0,021)$ y el diagnóstico de ingreso $(\mathrm{p}=0,013)$. Por el contrario, no existen diferencias significativas según lugar de residencia $(\mathrm{p}=0,309)$. 


\section{TABLA I}

DISTRIBUCIÓ N DE LA M UESTRA SEGÚN HÁBITO DE FUM AR, EDAD, GÉNERO, EDUCACIÓ N, NIVEL SO CIO ECO NÓ M ICO, LUGAR DE RESIDENCIA Y DIAGNÓ STICO DE INGRESO. HOSPITAL UNIVERSITARIO VIRGEN M ACARENA, SEVILLA, 2001

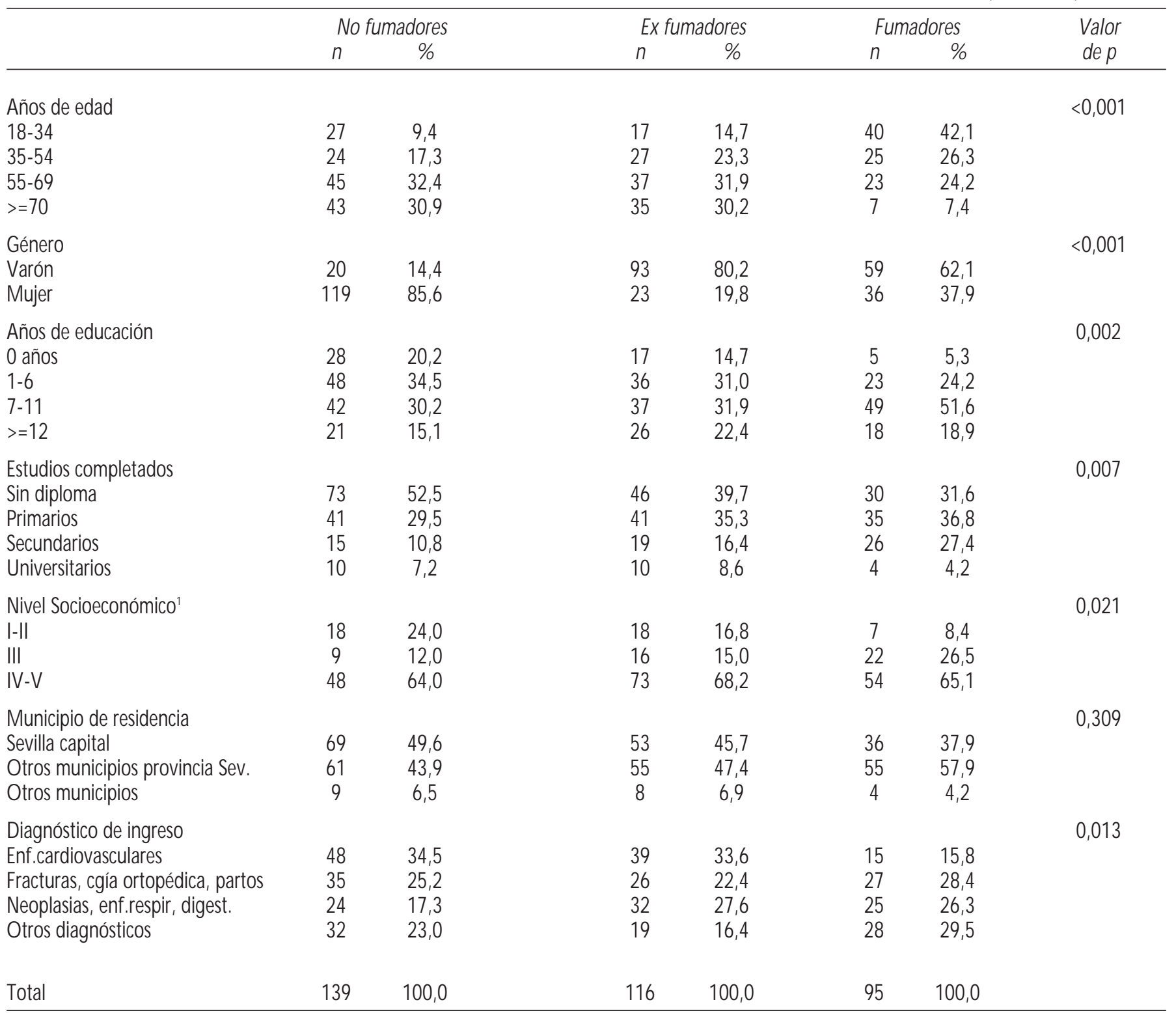

${ }^{\circledR}$ Los estratos no alcanzan el número total de pacientes por falta de información respecto a las amas de casa.

Los fumadores actuales son mayoritariamente jóvenes (42,1\% en $18-34$ años de edad), varones $(62,1 \%)$, han recibido entre 7 y 11 años de educación $(51,6 \%)$, tienen diploma de estudios primarios $(36,8 \%)$ y su principales motivos de ingreso son patologías agudas ( $28 \%$ con fracturas, cirugía ortopédica y partos). Por el contrario, los pacientes no fumadores tienen más edad $(63,3 \% \geq 55$ años), son mujeres $(85,6 \%)$, han recibido entre 1 y 6 años de educación $(34,5 \%)$, no han obtenido ningún certificado de nivel educativo completado $(52,5 \%)$ y su principal motivo de ingreso son las enfermedades cardiovasculares $(34,5 \%)$.

La distribución según nivel socioeconómico es más favorable a los no fumadores con un $24 \%$ en el estrato que agrupa a los niveles I y II que a los ex fumadores (16,8\% en I-II) y los fumadores actuales $(8,4 \%$ en I-II). Las enfermedades cardiovasculares son el motivo de ingreso de porcentajes similares de ex fumadores $(33,6 \%)$ y no fumadores $(34,5 \%)$ y un porcentaje más bajo de fumadores actuales $(15,8 \%)$. El grupo de enfermedades respiratorias, digestivas y neoplasias suma un $17,3 \%$ en no fumadores, en contraste con el $27,6 \%$ de ex fumadores y el $26,3 \%$ de fumadores actuales.

La tabla II muestra los conocimientos de los pacientes respecto a los efectos sobre la salud del hábito de fumar. Más del $90 \%$ de los pacientes entrevistados conocen que el tabaquismo causa cáncer de pulmón $(91 \%)$ y enfermedad pulmonar obstructiva crónica $(92 \%)$, pero los porcentajes se reducen 


\section{TABLA ॥}

CONO CIM IENTO DE LOS EFECTOS SO BRE LA SALUD DEL HÁBITO DE FUM AR. HOSPITAL UNIVERSITARIO VIRGEN M ACARENA, SEVILLA, 2001

\begin{tabular}{|c|c|c|c|c|}
\hline Fumar causa... & $\begin{array}{l}\text { No fumadores } \\
n \%\end{array}$ & $\begin{array}{l}\text { Ex fumadores } \\
n \%\end{array}$ & $\begin{array}{c}\text { Fumadores } \\
n \%\end{array}$ & $\begin{array}{c}\text { Total } \\
n \%\end{array}$ \\
\hline $\begin{array}{l}\text {... cáncer de pulmón? } \\
\text { Sí } \\
\text { No } \\
\text { No sabe } \\
\text { Valor de } p^{1}\end{array}$ & $\begin{array}{c}128(92,1) \\
2(1,4) \\
9(6,5)\end{array}$ & $\begin{array}{c}103(88,8) \\
2(1,7) \\
11(9,5)\end{array}$ & $\begin{array}{c}86(90,5) \\
3(3,2) \\
6(6,3)\end{array}$ & $\begin{array}{c}317(90,6) \\
7(2,0) \\
26(7,4) \\
0,376\end{array}$ \\
\hline $\begin{array}{l}\ldots . . \text { enf pulm obst crón? } \\
\text { Sí } \\
\text { No } \\
\text { No sabe } \\
\text { Valor de } p^{1}\end{array}$ & $\begin{array}{c}129(92,8) \\
0(0,0) \\
10(7,2)\end{array}$ & $\begin{array}{c}105(90,5) \\
1(0,9) \\
10(8,6)\end{array}$ & $\begin{array}{c}87(91,6) \\
3(3,2) \\
5(5,3)\end{array}$ & $\begin{array}{c}321(91,7) \\
4(1,1) \\
25(7,1) \\
0,031\end{array}$ \\
\hline $\begin{array}{l}\ldots . . \text { enf cardiovascular? } \\
\text { Sí } \\
\text { No } \\
\text { No sabe } \\
\text { Valor de } \mathrm{p}^{1}\end{array}$ & $\begin{array}{c}114(82,0) \\
2(1,4) \\
23(16,5)\end{array}$ & $\begin{array}{c}97(83,6) \\
5(4,3) \\
14(12,1)\end{array}$ & $\begin{array}{c}77(81,1) \\
4(4,2) \\
14(14,7)\end{array}$ & $\begin{array}{c}288(82,3) \\
11(3,1) \\
51(14,6) \\
0,209\end{array}$ \\
\hline $\begin{array}{l}\text {... nacidos bajo peso? } \\
\text { Sí } \\
\text { No } \\
\text { No sabe } \\
\text { Valor de } \mathrm{p}^{1}\end{array}$ & $\begin{array}{c}111(79,9) \\
3(2,2) \\
25(18,0)\end{array}$ & $\begin{array}{c}88(75,9) \\
2(1,7) \\
26(22,4)\end{array}$ & $\begin{array}{c}73(76,8) \\
4(4,2) \\
18(18,9)\end{array}$ & $\begin{array}{c}272(77,7) \\
9(2,6) \\
69(19,7) \\
0,364\end{array}$ \\
\hline $\begin{array}{l}\text {... cancer oral? } \\
\text { Sí } \\
\text { No } \\
\text { No sabe } \\
\text { Valor de } p^{1}\end{array}$ & $\begin{array}{c}109(78,4) \\
5(3,6) \\
25(18,0)\end{array}$ & $\begin{array}{c}99(85,3) \\
2(1,7) \\
15(12,9)\end{array}$ & $\begin{array}{c}77(81,1) \\
3(3,2) \\
15(15,8)\end{array}$ & $\begin{array}{c}285(81,4) \\
10(2,9) \\
55(15,7) \\
0,735\end{array}$ \\
\hline $\begin{array}{l}\text {... cáncer laringe? } \\
\text { Sí } \\
\text { No } \\
\text { No sabe } \\
\text { Valor de } \mathrm{p}^{1}\end{array}$ & $\begin{array}{c}118(84,9) \\
1(0,7) \\
20(14,4)\end{array}$ & $\begin{array}{c}101(87,1) \\
3(2,6) \\
12(10,3)\end{array}$ & $\begin{array}{c}83(87,4) \\
2(2,1) \\
10(10,5)\end{array}$ & $\begin{array}{c}302(86,3) \\
6(1,7) \\
42(12,0) \\
0,396\end{array}$ \\
\hline $\begin{array}{l}\text {... cáncer esófago? } \\
\text { Sí } \\
\text { No } \\
\text { No sabe } \\
\text { Valor de } p^{1}\end{array}$ & $\begin{array}{c}118(84,9) \\
1(0,7) \\
20(14,4)\end{array}$ & $\begin{array}{c}101(87,1) \\
3(2,6) \\
12(10,3)\end{array}$ & $\begin{array}{c}82(86,3) \\
2(2,1) \\
11(11,6)\end{array}$ & $\begin{array}{c}301(86,0) \\
6(1,7) \\
43(12,3) \\
0,389\end{array}$ \\
\hline $\begin{array}{l}\text { ¿Son adictos físicos } \\
\text { a la nicotina los fumadores? } \\
\text { Sí } \\
\text { No } \\
\text { No sabe } \\
\text { Valor de } \mathrm{p}^{1}\end{array}$ & $\begin{array}{c}103(74,1) \\
5(3,6) \\
31(22,3)\end{array}$ & $\begin{array}{c}97(83,6) \\
5(4,3) \\
14(12,1)\end{array}$ & $\begin{array}{c}79(83,2) \\
6(6,3) \\
10(10,5)\end{array}$ & $\begin{array}{c}279(79,7) \\
16(4,6) \\
55(15,7) \\
0,474\end{array}$ \\
\hline Total & $139(100,0)$ & $116(100,0)$ & $95(100,0)$ & $350(100,0)$ \\
\hline
\end{tabular}

${ }^{1}$ Excluyendo los participantes que responden no sabe y aplicando un test de tendencia lineal ya que no se cumplen los requisitos de aplicación de la prueba de x cuadrado de Pearson.

cuando la pregunta se refiere a su relación causal con el cáncer de laringe $(86 \%)$, esófago $(86 \%)$, cavidad oral $(81 \%)$ y cardiopatía coronaria $(82 \%)$. Este porcentaje es especialmente bajo cuando se pregunta sobre la posible asociación causal entre el hábito de fumar de las madres gestantes y el bajo peso de los recién nacidos (78\%). 
Tan sólo el 79,9\% de los pacientes reconoce que los fumadores son adictos físicos a la nicotina, con porcentajes que oscilan entre el $74,1 \%$ en los no fumadores y el $83,6 \%$ y $83,2 \%$ en ex fumadores y fumadores actuales, respectivamente.

En todos los epígrafes de la tabla II el porcentaje de pacientes que responde "no se" es mayor que el de pacientes que responde "no", negando clara y marcadamente estas relaciones causales. No existen diferencias estadísticamente significativas entre las respuestas (afirmativas y negativas, tras excluir los que afirman "no conocer”la posible relación) en no fumadores, ex fumadores y fumadores actuales.

La tabla III muestra las percepciones y actuaciones respecto al hábito de fumar en los pacientes de la muestra según su hábito tabáquico. Únicamente el $29,7 \%$ de los pacientes piensa que el hábito de fumar está inversamente relacionado con el nivel de ingresos y el $97 \%$ de los pacientes reconoce que abandonar el hábito de fumar es beneficioso incluso en los mayores de 60 años de edad $(97,1 \%$ ) y que el humo del tabaco de los fumadores perjudica a los no fumadores que conviven y trabajan con fumadores $(96,9 \%)$ no siendo significativas las diferencias en la distribución de estas respuestas según el hábito de fumar de los pacientes de la muestra.

Sí son significativas las diferencias entre no fumadores, ex fumadores y fumadores actuales respecto a las molestias que les produce el humo del tabaco (muy molesto para el 54,7\% de no fumadores versus $7,4 \%$ en fumadores), la delimitación de los espacios públicos en los que se debería poder fumar (en ningún área pública según el 28,1\% de no fumadores vs 7,4\% de fumadores), si ha rogado a alguien que no fume en su presencia $(54,0 \%$ de los no fumadores vs $13,7 \%$ de los fumadores) y si ha realizado algún intento para que se reduzca el humo de tabaco en su zona de trabajo (50\% de los no fumadores vs $11,3 \%$ de fumadores, tras excluir los pacientes sin empleo, pensionistas y quienes no recuerdan).

La tabla IV muestra algunas características del hábito de fumar y los intentos de deshabituación de los pacientes de la muestra que son fumadores así como algunas influencias del entorno familiar, social y sanitario sobre dichos pacientes.

La edad mas frecuente de comienzo del hábito de fumar es antes de los 15 años $(45,3 \%)$ y antes de cumplir los 20 años habían comenzado a fumar el 85,3\% de los mismos. La mitad de los fumadores enciende su primer cigarrillo en la primera hora después de levantarse y el $85 \%$ antes de que transcurran 3 horas. El 34,7\% de los fumadores consume más de una cajetilla diaria de cigarrillos y el $36,8 \%$ de los pacientes fumadores tienen una pareja que también fuma.

Sólo el 24,2\% no querría reducir el número de cigarrillos fumados; del resto, el 3,2\% declara no saber si desea reducir el tabaco y al 72,6\% le gustaría reducir los cigarrillos fumados en diferentes proporciones. Casi la mitad de los pacientes fumadores $(46,3 \%)$ ha intentado alguna vez, seriamente, dejar de fumar. Sin embargo, un $28,4 \%$ no confía nada en que podría dejar el hábito tabáquico.

El 37,9\% de los fumadores consiguió no fumar al menos durante 24 horas en el año anterior a su hospitalización actual. Un $11,6 \%$ lo hizo una vez, un $15,8 \%$ lo intentó entre 2 y 12 veces y un $10,5 \%$ dejó de fumar durante al menos 24 horas 13 o más veces en el último año.

El 29,5\% de los pacientes fumadores refieren que, durante el último año, una de las cinco personas más cercanas abandonó el hábito tabáquico y el 9,4\% contabilizan 2 o más perso- nas con igual conducta. El 36,8\% relatan que su familia y amigos le insisten "mucho" en que deje de fumar y el 61,1\% reconoce que en el último año ha recibido consejo sanitario de abandonar el hábito tabáquico.

\section{DISCUSIÓN}

La prevalencia de fumadores en nuestra muestra $(27,1 \%)$ es inferior a los valores nacional $(35,7 \%$ en 1997) y andaluz (38,2\% en 1996). Estas diferencias pueden estar parcialmente justificadas porque tanto la Encuesta Nacionales de Salud (27) como el Comisionado para la Droga (28), origen de las estimaciones citadas, miden prevalencia de tabaquismo en población $>=16$ años mientras que nuestro estudio se limita a pacientes $>=18$ años de edad. Por otra parte, la distribución por género muestra en los varones un $34,3 \%$ de fumadores (n $=59), 54,1 \%$ de ex fumadores $(n=93)$ y $11,6 \%$ de no fumadores $(\mathrm{n}=20)$ mientras que las mujeres se estratifican en $20,2 \%$ de fumadoras $(\mathrm{n}=36), 12,9 \%$ de ex fumadoras $(\mathrm{n}=$ $12,9)$ y $66,9 \%$ de no fumadoras $(n=119)$. Los porcentajes de fumadores son similarmente bajos por género en esta muestra hospitalaria, en comparación con los datos de prevalencia de tabaquismo en España en 1997, con $44,8 \%$ en varones y $27,2 \%$ en mujeres (27).

En nuestra muestra el porcentaje de fumadores decrece con la edad mientras aumenta el porcentaje de ex fumadores, relación inversa que se ha descrito también para los datos nacionales (27). De hecho, la mayoría de los fumadores abandonan el hábito después de 20-30 años de adicción (29).

La distribución conjunta, en nuestra muestra, de edad y hábito de fumar, agrupando en dos categorías: no fumadores versus fumadores -actuales y ex fumadores-, nos muestra una prevalencia, no puntual sino de periodo, del $60 \%$ de pacientes fumadores en algún momento de su vida, con un máximo homogéneo en los dos primeros grupos de edad, 18-34 y 3554 años, del 68\%, que desciende al 57\% en el grupo de 55-69 años y al $49 \%$ en los pacientes con 70 años y más. Esta distribución sugiere un efecto cohorte, con prevalencia mayor de hábito tabáquico -mantenido o no- en los pacientes nacidos en fechas más recientes y menor en los nacidos con anterioridad a 1945, pero a éste se sumaría un posible efecto de "selección natural" ya que el hábito de fumar se asocia con un incrementado riesgo de un amplio abanico de patologías graves y la reducción, paralela al envejecimiento, de una proporción de los fumadores por la propia mortalidad asociada al hábito de fumar es un hecho sanitario constatado.

Más aún si tenemos en cuenta que la distribución de la edad de comienzo del hábito de fumar en relación a la edad de los pacientes muestra diferencias significativas porque el $53 \%$ de los pacientes con 55 y más años de edad $(57 \%$ en $>=70$ años y $48 \%$ en 55-69 años) comenzaron a fumar antes de los 15 años mientras que el $54 \%$ de los pacientes con menos de 55 años (61\% de 18-34 años y 46\% de 35-54 años) comenzaron más tardíamente, entre los 15 y 19 años de edad, siendo por tanto la media de exposición de las cohortes de más edad, mas larga y mayor la probabilidad de aparición de patologías asociadas.

Las cifras más bajas de fumadores se encuentran en los niveles socioeconómicos más altos (I y II) en los que de 43 participantes sólo 7 son fumadores $(16,3 \%)$, pero las cifras más elevadas de fumadores no corresponden a las clases 
TABLA III

PERCEPCIO NES Y ACTUACIO NES RESPECTO AL HÁBITO DE FUMAR. HOSPITAL UNIVERSITARIO VIRGEN MACARENA, SEVILLA, 2001

Piensa que...
... fuman más quienes
tienen menos ingresos?

tienen menos ingresos?

Sí

No

No sabe

Valor de $p^{1}$

... dejar de fumar es beneficioso 2 ?

Sí

No

No sabe

Valor de $\mathrm{p}^{1,3}$

... los fumadores deberían fumar...

En cualquier área pública

En áreas de fumadores

En ningún área pública

No sabe/ no contesta

Valor de $p^{1}$

$47(33,8)$

$60(43,2)$

$32(23,0)$

$135(97,1)$

$1(0,7)$

$3(2,2)$

$4(2,9)$

$96(69,1)$

$39(28,1)$

$0(0,0)$

... el humo del tabaco perjudica

0 no a quienes no fuman..."?

Sí

No

No sabe

Valor de $\mathrm{p}^{1,3}$

... el humo de otros fumadores es...?

$\begin{array}{lc}\text { M uy molesto } & 76(54,7) \\ \text { Molesto } & 47(33,8) \\ \text { Nada molesto } & 15(10,8) \\ \text { No sabe/ no contesta } & 1(0,7)\end{array}$

Valor de $p^{1}$

¿En el pasado año....

... ha rogado a alguien que

no fumara en su presencia?

Sí

No

No recuerda

$75(54,0)$

$63(45,3)$

$1(0,7)$

$136(97,8)$

$3(2,2)$

$0(0,0)$

Valor de $p^{1}$

... ha intentado que se reduzca

el humo de tabaco

en su zona de trabajo?

Sí

No

No se fuma en su trabajo

Sin empleo / pensionista

No recuerda

Valor de $p^{5}$
$33(28,4)$

$58(50,0)$

$25(21,6)$

$112(96,6)$

$3(2,6)$

$1(0,9)$

$8(6,9)$

$83(71,6)$

$25(21,6)$

$0(0,0)$

$(7,4)$

$80(84,2)$

$7(7,4)$

$1(1,1)$

$3(97,9)$

$1(1,1)$

$1(1,1)$

$5(1,4)$

$5(1,4)$

0.735

$19(5,4)$

$259(74,0)$

$71(20,3)$

$1(0,3)$

0.002

$112(96,6)$

$1(0,9)$

$3(2,6)$

$49(42,2)$

$41(35,3)$

$26(22,4)$

$0(0,0)$

$91(95,8)$

$4(4,2)$

$339(96,9)$

$0(0,0)$

$8(2,3)$

$3(0,9)$

0.375

$7(7,4)$

$132(37,7)$

$29(30,5)$

$59(62,1)$

$0(0,0)$

$117(33,4)$

$100(28,6)$

$1(0,3)$

$<0.001$
$13(13,7)$

$82(86,3)$

$0(0,0)$

$136(38,9)$

$213(60,9)$

$1(0,3)$

$<0.001$
$15(10,9)$
$15(10,9)$
$7(5,1)$
$101(73,2)$
$1(0,7)$

139 (100)
$9(7,8)$

$25(21,6)$

$4(3,4)$

$78(67,2)$

$0(0,0)$
$6(6,3)$

$47(49,5)$

$3(3,2)$

$39(41,1)$

$0(0,0)$
$30(8,6)$

$87(24,9)$

$14(4,0)$

$218(62,5)$

$1(0,3)$

0.001

Total

116 (100)

95 (100)

350 (100)

${ }^{1}$ Excluyendo quienes responden no sabe, no contesta. ${ }^{2}$ ncluso en edades superiores a los 60 años. ${ }^{3}$ Test de tendencia lineal, ya que no se cumplen los requisitos de aplicación de la prueba de $x$ cuadrado de Pearson. ${ }^{4} Y$ conviven 0 trabajan con fumadores. ${ }^{5}$ Excluyendo los participantes en cuyos trabajos no se fuma, quienes están sin empleo 0 son pensionistas y el que responde que no recuerda. 
HÁBITO DE FUMAR, INTENTOS DE DESHABITUACIÓ N E INFLUENCIAS DEL ENTO RNO DE LOS PACIENTES FUM ADO RES. HOSPITAL UNIVERSITARIO VIRGEN M ACARENA, SEVILLA, 2001

\begin{tabular}{|c|c|c|}
\hline & Número & Porcentaje \\
\hline $\begin{array}{l}\text { Edad de comienzo del hábito de fumar } \\
<15 \text { años } \\
15-19 \text { años } \\
>=20 \text { años }\end{array}$ & $\begin{array}{l}43 \\
38 \\
14\end{array}$ & $\begin{array}{l}45,3 \\
40,0 \\
14,7\end{array}$ \\
\hline $\begin{array}{c}\text { Intervalo medio desde despertar hasta fuma } 1 \text { cigarrillo } \\
\qquad 1 \text { hora } \\
1-3 \text { horas } \\
>=4 \text { horas }\end{array}$ & $\begin{array}{l}48 \\
33 \\
14\end{array}$ & $\begin{array}{l}50,5 \\
34,7 \\
14,7\end{array}$ \\
\hline $\begin{array}{l}\text { Consumo medio actual de cigarrillos } \\
\qquad=10 \\
11-20 \\
>=21\end{array}$ & $\begin{array}{l}37 \\
25 \\
33\end{array}$ & $\begin{array}{l}38,9 \\
26,3 \\
34,7\end{array}$ \\
\hline $\begin{array}{l}\text { ¿Q ué hábito de fumar tiene su pareja? } \\
\text { No fumador } \\
\text { Ex fumador } \\
\text { Fumador } \\
\text { Sin pareja actual }\end{array}$ & $\begin{array}{c}35 \\
6 \\
35 \\
10\end{array}$ & $\begin{array}{r}36,8 \\
6,3 \\
36,8 \\
20,0\end{array}$ \\
\hline $\begin{array}{l}\text { ¿Q uerría reducir el número de cigarrillos que fuma y cuan } \\
\text { No quiero reducir el número de cigarrillos fumados } \\
\text { Si, en } 10 \text { cigarrillos } \\
\text { Si, en } 20 \text { cigarrillos } \\
\text { No sabe }\end{array}$ & $\begin{array}{c}23 \\
14 \\
55 \\
3\end{array}$ & $\begin{array}{c}24,2 \\
14,7 \\
57,9 \\
3,2\end{array}$ \\
\hline $\begin{array}{c}\text { ¿Ha intentado alguna vez seriamente dejar de fumar? } \\
\text { Sí } \\
\text { No }\end{array}$ & $\begin{array}{l}44 \\
51\end{array}$ & $\begin{array}{l}46,3 \\
53,7\end{array}$ \\
\hline $\begin{array}{l}\text { ¿Cuánto confía en que podría dejar de fumar? } \\
\text { No confío nada } \\
\text { Confío algo } \\
\text { Estoy muy confiado } \\
\text { No sabe }\end{array}$ & $\begin{array}{c}27 \\
29 \\
36 \\
3\end{array}$ & $\begin{array}{c}28,4 \\
30,5 \\
37,9 \\
3,2\end{array}$ \\
\hline $\begin{array}{l}\text { ¿Cuántas veces el año pasado dejó de fumar } 24 \text { horas }^{12} \\
\text { Ninguna } \\
1 \text { vez } \\
2-12 \text { veces } \\
>=13 \text { veces } \\
\text { No recuerda }\end{array}$ & $\begin{array}{l}42 \\
11 \\
15 \\
10 \\
17\end{array}$ & $\begin{array}{l}44,2 \\
11,6 \\
15,8 \\
10,5 \\
17,9\end{array}$ \\
\hline $\begin{array}{l}\text { ¿De las } 5 \text { personas más cercanas, cuantas dejaron de fum } \\
\text { Ninguna } \\
1 \text { persona } \\
>=2 \text { personas }\end{array}$ & $\begin{array}{c}58 \\
28 \\
9\end{array}$ & $\begin{array}{l}61,1 \\
29,5 \\
9,4\end{array}$ \\
\hline $\begin{array}{c}\text { ¿Cuánto le insisten su familia y amigos en que deje de fum } \\
\text { Prácticamente nada } \\
\text { Algo } \\
\text { Mucho } \\
\text { No sabe }\end{array}$ & $\begin{array}{l}15 \\
22 \\
35 \\
23\end{array}$ & $\begin{array}{l}15,8 \\
23,2 \\
36,8 \\
24,2\end{array}$ \\
\hline $\begin{array}{c}\text { ¿Le ha aconsejado personal sanitario que deje de fumar } \\
\text { Sí } \\
\text { No } \\
\text { No recuerda }\end{array}$ & $\begin{array}{c}58 \\
36 \\
1\end{array}$ & $\begin{array}{l}61,1 \\
37,9 \\
1,1\end{array}$ \\
\hline Total & 95 & 100,0 \\
\hline
\end{tabular}

Durante al menos 24 horas. ${ }^{2}$ Durante el último año. 
sociales IV y V sino al estrato socioeconómico intermedio (III). Estos resultados podrían verse influidos por la ausencia de información sobre ocupación de los cónyuges de las mujeres no activas, que ha impedido clasificar a éstas $(n=85)$ en ninguno de estos estratos. Sin embargo, este hecho debería afectar en mayor medida a las estimaciones por clase social en pacientes no fumadores, en los que el $85,6 \%$ son mujeres. Por otra parte, también podría reflejar una situación tabáquica de transición, con un elevada prevalencia global del hábito de fumar y una reciente y creciente oposición a dicho hábito en las clases socioeconómicas con mayor disponibilidad económica y cultural (10). De hecho, la distribución de fumadores según años de educación y máximo nivel educativo alcanzado parece apoyar esta última explicación ya que, en cada estrato, la proporción de fumadores aumenta progresivamente con los años de educación y el nivel más alto de educación completada excepto en los universitarios y quienes han cursado 12 o más años de estudios. Así, de 60 pacientes con estudios secundarios, el 43,3\% son fumadores $(n=26)$ mientras que de los 24 universitarios entrevistados sólo 4 fumaban $(16,7 \%)$.

Los resultados del estudio muestran que una gran mayoría de los pacientes conocen los efectos perjudiciales del tabaco sobre la salud, pero que una proporción de éstos aún ignora algunas relaciones causales bien establecidas. Sin embargo, este desconocimiento no muestra diferencias significativas según el estatus de hábito tabáquico y el porcentaje de pacientes que responde "no se" es siempre mayor que los que responden "no". Estos hallazgos parecen indicar que el conocimiento no influye sobre el hábito de fumar o, lo que parece más probable si tenemos en cuenta la edad temprana de comienzo del mismo, que existe un desfase entre conocimiento y hábito tabáquico; es decir, que el hábito de fumar se adquiere antes que el conocimiento o el reconocimiento de los efectos perjudiciales sobre la salud del mismo. Posiblemente, el fumar sea para algunos fumadores una de las pocas estrategias personales disponibles para afrontar el estrés, hecho que le confiera ventajas que, al menos en etapas tempranas del hábito sobresalgan sobre sus efectos negativos en la salud, que serán reconocidos más tarde, con la aparición de síntomas o por la observación en otros de los efectos adversos del tabaco (29)

Los no fumadores parecen haber desarrollado una actitud mas activa contra el hábito de fumar de lo que era habitual hasta recientemente. De hecho, respecto a los criterios de delimitación de las áreas para fumadores, la valoración de lo molesto del humo de otros fumadores y los intentos de evitarlo, en general, y particularmente en el trabajo, sí que existen diferencias significativas. Este hallazgo es razonable puesto que los no fumadores no encuentran ninguna ventaja del hábito de fumar y son cada vez más conscientes de las desventajas de su exposición al humo de tabaco como fumadores pasivos.

El corto intervalo de tiempo referido entre el despertar de los pacientes fumadores y el consumo del primer cigarrillo no parece concordar con el relativamente bajo consumo medio declarado de cigarrillos. La mitad de los fumadores tarda menos de 1 hora en fumar su primer cigarrillo pero sólo el $35 \%$ reconoce fumar más de un paquete diario. Una disparidad similar se produce al comparar esta última cifra con la del $58 \%$ de fumadores que desean reducir en 20 cigarrillos la cantidad fumada. Estos datos parecen indicar una subestimación en el consumo declarado de cigarrillos, posiblemente originada por el deseo o la necesidad de una proporción importante de fumadores de reducir o abandonar el hábito de fumar, sin conseguirlo.

De hecho, casi la mitad de los fumadores ha intentado alguna vez, seriamente y sin éxito, dejar de fumar y el 10,5\% dejó de fumar durante al menos 24 horas, más de una vez al mes. Sin embargo, casi dos tercios de los fumadores reconocen haber recibido consejo médico de abandonar el tabaquismo y a un $60 \%$ la familia y los amigos le insisten en que deje de fumar.

Todos estos datos apuntan hacia la existencia de una importante proporción de fumadores que necesitan ayudas y soportes adicionales para abandonar su hábito. Los métodos farmacológicos específicos para el tratamiento de la adicción tabáquica (30), que complementados con el seguimiento sanitario individualizado de los pacientes y el aprendizaje, por parte de éstos, de comportamientos para evitar el consumo, han producido tasas de abandono (12) modestas pero efectivas del 20\% deberían tenerse en cuenta dentro de la oferta terapéutica habitual, como ya se ha hecho en otros países de nuestro entorno con el objetivo de reducir o mantener descensos previos en las cifras de prevalencia de fumadores (31).

Los conocimientos respecto a los efectos prejudiciales del tabaco sobre la salud son similares en no fumadores, ex fumadores y fumadores, animando a una proporción de estos últimos a abandonar su hábito. Sin embargo, un porcentaje importante de fumadores intenta repetidamente deshabituarse sin éxito. Por tanto, debería ofertarse sistemáticamente apoyo sanitario -farmacológico y conductual- a quienes fuman y desean abandonar dicho hábito y no sólo recomendar el cese en el tabaquismo, ya que superar su adicción parece ser un gran problema para una proporción importante de fumadores.

\section{Bibliografía}

1. Fernández E, Schiaffino A, García M, Salto E, Villalbi JR, Borras JM. Prevalencia del consumo de tabaco en España entre 1945 y 1995. Reconstrucción a partir de las Encuestas Nacionales de Salud. Med Clin (Barc) 2003; 120: 14-6.

2. Tyczynski JE, Bray F, Parkin DM. Lung cancer in Europe in 2000: epidemiology, prevention, and early detection. Lancet Oncol 2003; 4: 4555.

3. Conway DI, Macpherson LM, Gibson J, Binnie VI. Oral cancer: prevention and detection in primary dental healthcare. Prim Dent Care 2002; 9: 119-23.

4. Menezes AM, Horta BL, Oliveira AL, Kaufmann RA, Duquia R, Diniz A, et al. Attributed risk to smoking for lung cancer, laryngeal cancer and esophageal cancer. Rev Saude Publica 2002; 36: 129-34.

5. Konner J, O'Reilly E. Pancreatic cancer: epidemiology, genetics, and approaches to screening. Oncology (Huntingt) 2002; 16: 1615-22.

6. Orth SR, Ritz E. The renal risks of smoking: an update. Curr Opin Nephrol Hypertens 2002; 11: 483-8.

7. Vaqué Rafart J, Almaraz Gómez A, Rosselló Urgell J. Tabaco y Salud. En: Gálvez R, Sierra A, Sáenz MC, Fernández-Crehuet, Salleras L, Cueto A, et al. Medicina Preventiva y Salud Pública. 10 a edición. Salvat. Barcelona, 2001.

8. Newburn-Cook CV, White D, Svenson LW, Demianczuk NN, Boff N, Edwards J. Where and to what extent in prevention of low birth weight possible? West J Nurs Res 2002; 24: 887-904. 
9. To Figueras J. Tabaco y cancer: de la asociación epidemiológica a la evidencia molecular. Med Clin (Barc) 1999; 112: 589-94.

10. Villalbí JR. El tabaco como problema de salud pública. Sociedad Española de Salud Pública y Administración Sanitaria. Informe SESPAS 2002: La salud y el sistema sanitario en España. Invertir en Salud. Prioridades para la salud pública en España. www.sespas.aragob.es/informe.html.

11. Ministerio de Sanidad y Consumo y Organización Mundial de la Salud. Informe de la primera conferencia europea sobre política del tabaco: ¿Se puede lograr una Europa libre de tabaco? Madrid: OMS / MSC; 1992.

12. Grandes G, Cortada JM, Arrazola A. ¿Podemos ayudar a nuestros pacientes a dejar de fumar?: La experiencia del Programa de Abandono del Tabaco. Gac Sanit 2001; 15: 312-9.

13. González Enríquez J, Rodríguez Artalejo F, Martín Moreno JM, Banegas Banegas JR, Villar Alvarez F. Muertes atribuibles al consumo de tabaco en España. Med Clin (Barc) 1989; 92: 15-8.

14. Rodriguez Tapioles R, Pueyos Sánchez A, Bueno Cavanillas A, Delgado Rodríguez M, Galvez Vargas R. Proporción de enfermedad atribuible al tabaco en la provincia de Granada. Med Clin (Barc) 1994; 102: $571-4$

15. García A, Hernández I, Alvarez-Dardet C. El abandono del hábito tabáquico en España. Med Clin (Barc) 1991; 97: 161-5.

16. Peto R, López A, Boreham J, Thun M, Heath C. Mortality from tobacco in developed countries: indirect estimation from national vital statistics. Lancet 1992; 339: 1268-78.

17. Peto R, Lopez AD, Boreham J, Thun M, Heath CJr, Doll R. Mortality from smoking worldwide. Br Med Bull 1996; 52: 12-21.

18. Pierce JP, Fiore MC, Novotny TE, Hatziandreu EJ, Davis RM. Trends in cigarette smoking in the United States. Educational differences are increasing. JAMA 1989; 261:56-60.
19. Brownson RC, Jackson J, Wilkerson J, Davis JR, Owens NW, Fisher EB. Demographic and socioeconomic differences in beliefs about the health effects of smoking. Am J Public Health 1992; 82 (1): 99-103.

20. Muñoz N, Herrero R, Franceschi S. Molecular epidemiology of cancer of oral cavity and oropharynx: A multi-center case-control study protocol. Lyon: International Agency for Research on Cancer; 1996.

21. Servicio Andaluz de Salud. Conjunto Mínimo Básico de Datos de Altas Hospitalarias 1999. Sevilla: SAS; 2001.

22. Clasificación Estadística Internacional de Enfermedades y Problemas relacionados con la Salud. Décima Revisión. Washington: OPS, 1995.

23. Alvarez-Dardet C, Alonso J, Domingo A, Regidor E. La medición de la clase social en Ciencias de la Salud. Informe de un grupo de trabajo de la Sociedad Española de Epidemiología. Barcelona: SG-Editores, 1995.

24. SPSS Advanced Statistics. SPSS inc. Chicago, 1999.

25. Armitage P, Berry G. Estadística para la investigación biomédica. $3^{\mathrm{a}}$ edición. Madrid: Harcourt Brace, 1997.

26. Domenech Massons JM. Fundamentos de Diseño y Estadística. Unidad didáctica 10. Relación entre dos variables categóricas: Pruebas de x cuadrado. Barcelona: Ed. Gráficas Signo; 2001.

27. Ministerio de Sanidad y Consumo. Tabaco. En: Ministerio de Sanidad y Consumo. Informe sobre la Salud de los Españoles, 1998; www.msc.es/salud/epidemiología/tabaco

28. Consejería de Salud. II Plan Andaluz de Salud. Sevilla: Junta de Andalucía, 1999.

29. Villalbí JR, Nebot M. Salud Pública y Tabaco. En: Martínez Navarro F, Castellanos Pl Gili M, Marset P, Navarro V. Salud Pública. Madrid: McGraw-Hill-Interamericana; 1999.

30. Cadime. Tabaquismo: tratamiento farmacológico. Boletín Terapéutico Andaluz 1996; 12: 21-2.

31. Chambers J. Being strategic about smoking. Br Med J 1999; 318: 1-2 